\title{
Performance of Random Contention PRMA: A Protocol for Fixed Wireless Access
}

\author{
Salman Ali AlQahtani ${ }^{1}$, Ahed Alshanyour ${ }^{2}$, Ashraf Mahmoud ${ }^{2}$ \\ ${ }^{1}$ Department of Information and Communications Technology, King Fahad Security College, Riyadh, Saudi Arabia \\ ${ }^{2}$ Computer Engineering Department, King Fahd University of Petroleum and Minerals, Dhahran, Saudi Arabia \\ E-mail:salman@kfsc.edu.sa, \{ahed,ashrafs\}@kfupm.edu.sa \\ Received May 21, 2011; revised June 24, 2011; accepted July 2, 2011
}

\begin{abstract}
This paper introduces a packet reservation multiple access (PRMA) with random contention for fixed wireless access communications. The performance of PRMA scheme with random contention is compared with the performances of traditional PRMA under permission contention scheme. The proposed scheme is a simpler contention mechanism that does not depend on a pre-determined permission probability as PRMA under permission contention scheme. In this new method, terminals select the contention slot uniformly from the pool of remaining free slots in the current frame. We evaluate the performance of the new contention mechanism in terms of various metrics including maximum number of carried voice calls and packet delays for a given acceptable drop rate of voice packets. We show that the new mechanism is superior to that of PRMA under permission contention scheme for loaded systems and is expected to be insensitive for traffic source burstiness.
\end{abstract}

Keywords: Contention Scheme, Fixed Wireless, Performance Evaluation, PRMA

\section{Introduction}

Packet reservation multiple access (PRMA) is designed for a two-way wireless communications network with a star topology. It enables dispersed terminals to transmit packetized information over a shred channel to a base station. While PRMA controls the upstream traffic, the base station broadcasts continuous binary feedback messages to the contended stations on each slot to indicate availability of these slots for contention for the subsequent frames. Due to this low control and feedback messages, PRMA is designed for indoor or short range microcell where the transmission delay are negligible and the feedback assumed to be available instantaneously.

In this paper we focus on voice transmission while PRMA could be used for integrated voice and data transmission. PRMA uses TDMA technology where transmission frame is divided into a set of slots. Terminals have packets for transmission contend on these slots. Successful contention leads to slot reservation but successful contention occurs only if one terminal contends on a given slot. Successful reservation for a slot is acknowledged by the base station. Collision occurs if more than one terminal contends for the same slot. Terminal that success in reserving a slot keeps on this reservation for transmitting subsequent packets, slot reservation is terminated as terminal buffer has no packets to transmit. To increase system efficiency PRMA uses speech activity detection. Speech activity detection is used at voice terminals to determine when the speaker is silent or talking.

Contention scheme in PRMA is based on permission probability, terminal that has packets to transmit can contend on the slot only if it has a permission to contend, this type of contention is used to avoid collision occurrence. For maximum system capacity, permission probability should be small because increasing this permission probability leads to excessive number of collisions. Also reducing the permission probability beyond a certain value leads to a poor utilization for free slots. For maximum system utilization, Nada and Goodman [1] use equilibrium point analysis to evaluate system behavior. For a particular example their numerical calculations show that the maximum capacity could be achieved with permission probability rages between 0.25 and 0.35 is 37 simultaneous conversations, these simultaneous conversations share a PRMA channel with 20 slots per frame [2]. 
All terminals mentor the binary feedback messages that are broadcasted by the base station. Based on these feedback messages terminals can know in advance the available slots within the transmission frame. Using permission scheme as proposed in [2], all active stations that have permission to transmit are contending on the first incoming available slot. This simultaneous contention will increase the probability of collision occurrence. In this work, we propose a new contention scheme that reduces the number of simultaneous contention on a specific available slot. The new proposed scheme, random contention scheme, is based on the fact that available slots are known in advance. Therefore, rather than allowing all ready active stations to contend on the incoming available slot, we limit this number of simultaneous contention by allowing each active terminal to select its expected available slot randomly from the pool of remaining free slots in the current frame. The new scheme still has the maximum capacity that is reached by the permission contention scheme.

This work is organized as follows. Section two is a survey of related work to PRMA. In Section three we described the PRMA technique as well as the new proposed contention scheme. In Section four, we present the simulation model used for performance analysis, and we discuss workload model and parameters used in the simulation. Also, the results of the performance evaluation are presented and discussed. Finally, conclusions are given in Section five.

\section{Related Works}

Contention techniques such as ALOHA and carrier sense multiple access (CSMA) are not suitable for serving large number of terminals. These techniques make inefficient use of the shared transmission medium. Moreover, with increasing number of terminals throughput goes down and transmission delay increases. [2] explored PRMA as a technique for transmitting over short range radio channels. The system is proposed as a solution for inefficient contention techniques.

There have been many proposals for enhancing the performance of PRMA which concentrated on improving channel efficiency more and providing some kind of fairness for data applications, neglecting the variable channel access delay problem [3-10]. Goodman et al. previous work extended in [3] by examining the influence of a large number of system variables on PRMA performance. Through computer simulation they found that with 32 kbps speech coding and $720 \mathrm{kbps}$ transmission medium PRMA can support up to 37 simultaneous conversations under dropping probability less than 0.01 . In [1], their previous work are extended by using equilibrium point analysis to evaluate PRMA system behavior mathematically. They derive the probability of packet dropping given the number of simultaneous conversations; they also establish conditions for system stability and efficiency. Their numerical calculation show close agreement with computer simulation results. In [4], it has been shown that PRMA gracefully accept low-rate data terminals with moderate data packet delays and extended the equilibrium point analysis by including voice and data traffic in the numerical model.

To prevent slots from being wasted in the event of a collision during access contention, [5] proposed a minislotted PRMA protocol. The proposed protocol is shown to yield improvements over PRMA for both types of traffic, voice and data, at the expense of introducing small amounts of additional clipping to some talk spurts. In an ideal condition with only speech traffic, the proposed system supports 41 speech terminals with less than $1 \%$ packets dropped. To overcome the stability problem under heavy load conditions, a modified version, which is referred to as non-collision PRMA, NC-PRMA, is proposed in [6]. The protocol is based on assigning a pair of dedicated control minislots in the call set-up phase for each terminal, the contention mechanism required for reservation in the PRMA protocol is replaced by an individual reservation procedure. As expected, packet collision never occurs. The study results also show that the overall performance of the NC-PRMA system is superior to that of the PRMA system.

Authors in [7] proposed a hybrid access protocol known, as contention time-division multiple access (CTDMA). C-TDMA shows some features of contentionbased (slotted-ALOHA) and reservation-based (PRMA) protocols. It has been recommended for use in the uplink of future European multimedia distribution systems. A simple Markov model is proposed to describe C-TDMA behavior. Their results in terms of throughput and delay under variable traffic conditions indicate that C-TDMA is able to grant optimum throughput/delay figures for typical multiuser systems. Moreover, for a digital speech scenario, a performance comparison with PRMA demonstrates that C-TDMA yields equivalent performance to PRMA in terms of number of users supported by the system with a limited packet dropping rate.

In this work, which is an extension of [9], we propose the use of a simple contention resolution method for voice terminals that does not depend on a pre-determined permission probability figure as in the original PRMA. In this method, the user selects a slot uniformly from the pool of remaining free slots in the frame. In the results section we show that the proposed method while produces the same capacity figures as the original method, provides lower packet delays for loaded systems. Finally, 
we expect the new method to be insensitive with respect to traffic burstiness degree.

\section{PRMA System Descriptions}

PRMA is a technique for transmitting, over short range radio channels, a mixture of voice and data packets. The PRMA protocol is organized around time frames with duration matched to the periodic rate of voice packets. In each frame, active terminals try to reserve free time slot dynamically. PRMA is closely related to reservation ALOHA, R-ALOHA. It is distinguished from R-ALOHA by its response to network congestion and by its short round-trip transmission time. In R-ALOHA, congestion causes excessive packet delays. In PRMA, information packets are discarded if they remain in terminal transmission buffer beyond a certain time limit. For indoor application the round-trip propagation time between terminals and base stations is less than one microsecond. Packet durations typically are $500-1000 \mu \mathrm{s}$. The short propagation times allow terminals to learn quickly the results of transmission attempts. In many cases, an acknowledgment message for the current time slot can arrive at the terminals before the beginning of the next time slot $[1,2]$.

PRMA is a slotted protocol where frame duration, $T$, is a design variable parameter divided into a set of slots, $\mathrm{N}$, number of these slots depends on both frame size and transmitted packet size. Frame size is related to frame duration and channel transmission rate, $R_{c}$. Packet size is related to source bit rate, $R_{s}$. Terminal generates only one speech packet during frame time. The amount of source information per packet is $R_{s} T$ bits and the total length is $R_{s} T+H$ bits. $H$ represents the size of the header. Header contains routing, synchronization and control information.

$$
N=\left[\frac{R_{c} T}{R_{c} T+H}\right]
$$

The speech activity detector is modeled as a two-state Markov process as shown in Figure 1.

The probability of transmission from the talk, TLK, state toward the silence state, SIL, is the probability that the talkspurt with mean duration $\mathrm{t} 1$ ends during a time

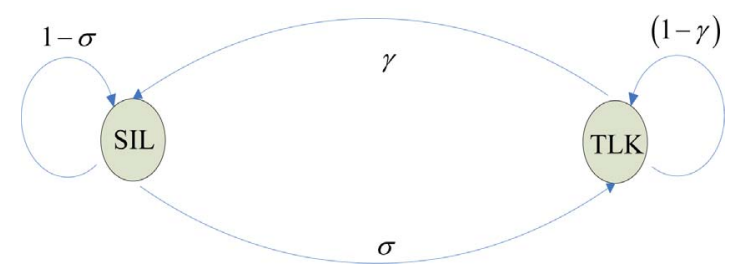

Figure 1. Two-state model of a slow speech activity detector. States are talking (TLK) and silence (SIL). slot of duration $\tau$.

$$
\gamma=1-\exp \left(-r / t_{1}\right)
$$

While the probability of transmission from the SIL state to the TLK state is the probability that silencespurt with mean duration $t_{2}$ ends during a time slot $\tau$

$$
\sigma=1-\exp \left(-\tau / t_{2}\right)
$$

Figure 2 represents a PRMA speech terminal with $N+$ 2 states. Terminal could be in one of the $N+2$ states, speech activity detector control the transmission of the terminal between SIL state and contending state, CON. Detecting talkspurt moves the terminal toward CON state and terminal starts contending on available time slots as packets be ready for transmission in its transmitting buffer. Detecting silencespurt by speech activity detector moves the terminal into the silence state.

The terminal in CON state gets a reservation and start transmit if the following three conditions are hold simultaneously: 1)The contended slot is unreserved; the probability that the slot is not reserved is $(1-r)$, where $\mathrm{r}$ is the probability that the slot is reserved by some other terminal. 2) The terminal has a permission to transmit, transmission permission is $p$. 3) No other terminals have a permission to transmit $u=(1-p)^{c-1}$, where $c$ represent the number of contended terminals.

Permission for transmission is generated according to a binary random event generator independently at each terminal. The permission probability, $p$, is a system design parameter. A feedback message from the central base station is broadcasted to all terminals with the end of each time slot to indicate whether this slot is reserved or still available for reservation in the next frame. By the starting of each slot, terminal that has packets and permission start transmitting their first available packet. Base station detects collision if it gets a disturbed signal due to the transmission of two packets or more within the

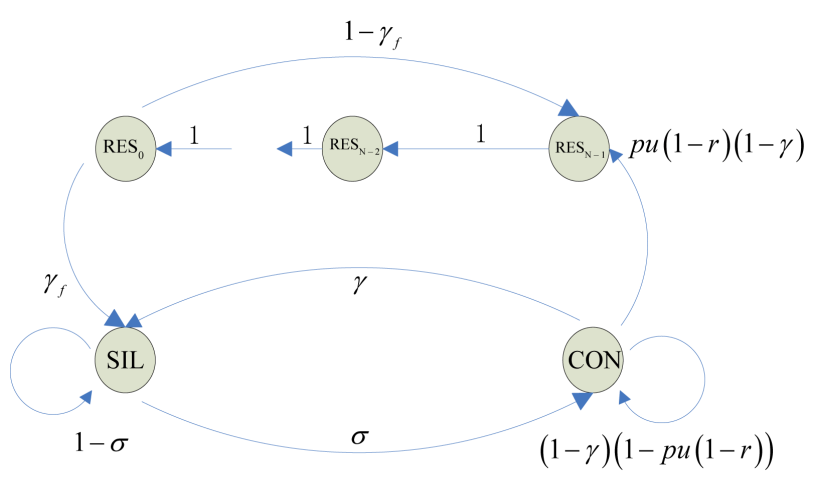

Figure 2. Transition State Diagram for Voice Terminals, $\gamma_{f}$, represents the probability of transition from state RESO to state SIL, this probability is equal the probability that the talkspurt ended in the most recent frame. 
same time slot. Terminals can detect whether they encounter a collision or no by detecting the feedback message broadcasted by the base station. If some terminal encounters a collision it keeps the transmitted packet in its buffer and tries to contend on other available time slot. Terminal that succeeded in reserving the contended time slot keeps on this reservation for transmitting all subsequent speech packets it generates during the talking spurt.

In our new contention scheme, terminal with ready packets to transmit select their slots by running a uniform random generator on available free slots. Collision could occur if more than one terminal contends randomly for the same time slot. Terminal starts transmitting its speech packets as the contended slot is coming. Collision detected by the terminal after receiving the feedback broadcasted message from the base station. Collided terminals start picking new available slots for transmission. This scheme of contention could increase packet queuing delay by deferring packet transmission until the availability of the contended slot, but it reduces the number of collisions compared with the permission contention scheme. Reducing number of collisions will reduce packet queuing delay for heavy traffic systems, because in these systems the probability of contending on the same slot by more than one terminal is relatively high. This high probability induces successive collisions for incoming slots. Reducing this probability requires reducing permission probability, but reducing permission probability beyond a certain value results in wasting many available time slots.

Speech packets are sensitive to the delay; therefore, speech packets cannot tolerate high queuing delay, a maximum threshold delay, $D_{\max }$ is considered for packets. Packets with queuing delay exceeding this threshold delay dropped from terminal transmitter buffer. Packets transmission depends on slot reservation; therefore packets could encounter excessive queuing delay. Terminals transmitter buffers start dropping the oldest packets if there is no available space for recent generated packets. Buffer size, B, depends on both the maximum threshold delay and the frame duration.

$$
B=\left\lceil\frac{D_{\max }}{T}\right\rceil
$$

\section{Silulation Results}

A simulation program is written to evaluate the efficiency of PRMA under the new contention scheme compared against the permission contention scheme. Table 1 provides a summary of system parameters that are used in our simulation program. These parameter values are adopted in [2] study for evaluating PRMA efficiency under permission contention scheme.

The number of simultaneous conversations has been used as a running parameter in all experiments; the maximum number of simultaneous conversations that can take place with $p_{\text {drop }} \leq 0.01$ is adopted as a measure of system capacity, $M . p_{\text {drop }}$ is defined as the number of dropped packets to total number of generated packets. The Effect of different system parameters on PRMA with random contention scheme are evaluated and compared with PRMA under permission contention scheme.

With our new proposed contention scheme, terminals always have permission for transmission. While in permission contention scheme terminals are allowed to contend on the available slots only if they have permission to do that, this permission is generated in each terminal independently from other terminals. Figure 3 shows that with permission scheme the maximum number of simultaneous conversations is changed with changing permission probability.

Table 1. PRMA variables.

\begin{tabular}{cccc}
\hline Parameter & Notation & Units & Values \\
\hline Talk Spurt Mean Duration & $t_{1}$ & $\mathrm{sec}$ & 1.0 \\
Silent Spurt Mean Duration & $t_{2}$ & $\mathrm{sec}$ & 1.35 \\
Channel Rate & $R_{\mathrm{c}}$ & $\mathrm{b} / \mathrm{s}$ & 720000 \\
Source Rate & $R_{\mathrm{s}}$ & $\mathrm{b} / \mathrm{s}$ & 32000 \\
Frame Duration & $T$ & $\mathrm{sec}$ & 0.016 \\
Overhead & $H$ & $\mathrm{bit}$ & 64 \\
Speech Activity Detector & Slow & & \\
Maximum Delay & $D_{\max }$ & $\mathrm{sec}$ & 0.032 \\
Dropping Probability & $p_{d r o p}$ & & 0.01 \\
Permission Probability & $p$ & & 0.3 \\
Conversations & $M$ & & Variable \\
\hline
\end{tabular}

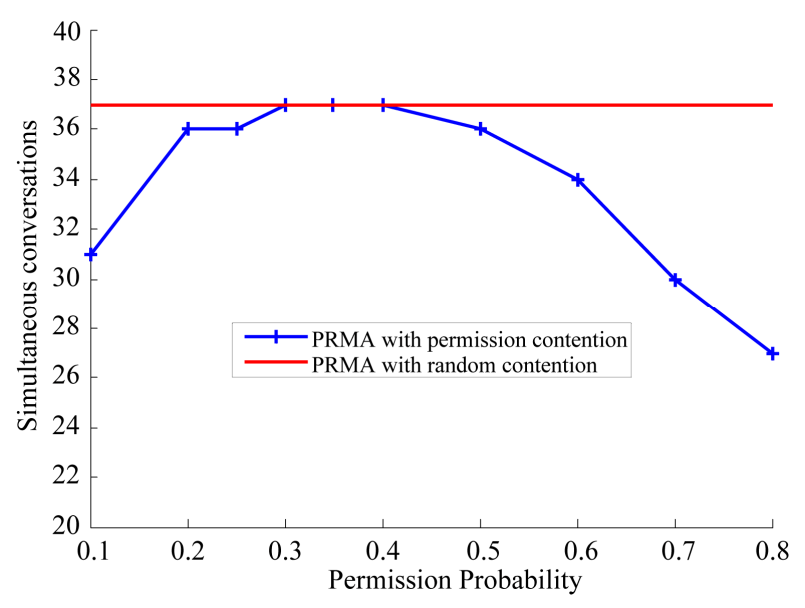

Figure 3. Maximum number of simultaneous conversation with $p_{\text {drop }} \leq \mathbf{0 . 0 1}$ as a function of permission probability, $p$. 
For both low and high permission probability, most of available slots are wasted without reservation. With low permission probability slots are wasted because terminals have no permission for contention while with high permission probability, slots are wasted due to collision between contended stations, the maximum number of simultaneous conversations reached is $M=37$ when $p=$ 0.3 . Using our new approach give us the same maximum number of simultaneous conversations. Our approach is better than the permission approach in terms of the average packet delay under heavy load traffic and number of collisions in light load traffic. Figure 4 and Figure 5 show these facts. Packet delay is considered as the elapsed time between packet generation and the start of packet transmission, taking in account the limitation on this time it should not exceed the maximum packet delay, $D_{\max }$. Figure 4 shows that for maximum number of simultaneous conversations the average packets delay is less than that in permission contention scheme. The high values for the average packet delay under light load traffic are due to the nature of random contention scheme. In random contention scheme terminals could contend on any available slot from the pool of remaining free slots in the current frame, this slot could be just the next one or could be the last one in the frame, in average terminals need to wait for $4 \mathrm{msec}$ (frame duration, $T=16 \mathrm{~ms}$ ). The low average packet delay at high traffic load is good because the objective from exploring PRMA system is to achieve high capacity with minimum packet delay.

Figure 5 shows the number of collisions as a function of the number of simultaneous conversations. The number of collisions is increased with increasing the traffic load due to the possibility of contending on the same slot by many terminals at the same time. Permission contention scheme solved this problem by reducing the permission probability. Our new proposed scheme reduces the number of collisions by distributing slots contention

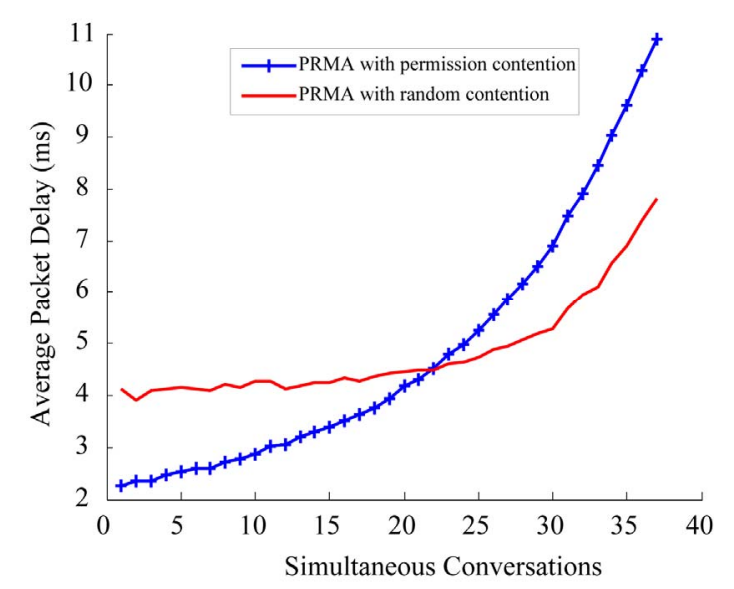

Figure 4. Packet average delay $p_{\text {drop }} \leq \mathbf{0 . 0 1}$ as a function of number of simultaneous conversations.

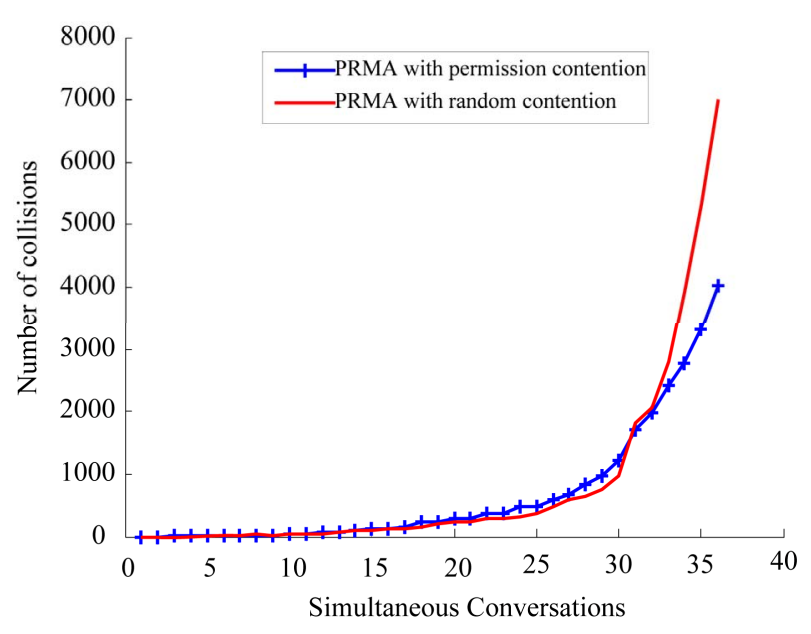

Figure 5. Number of collisions with $p_{\text {drop }} \leq 0.01$ as a function of simultaneous conversations.

among all available slots rather than contending on the same slot. Still our scheme faces collisions due to the possibility of selecting randomly the same available slot by different terminals. But the occurrence number of these collisions in low traffic case is less than those in permission contention scheme. In case of high traffic load the number of collisions starts increasing rapidly because all terminals that have data to transmit try to contend randomly on very little number of slots. A solution for this problem could be by adopting a dynamic contention scheme that reduces the number of collisions in case of low number of available slots.

The frame duration, $T$, is determined by the amount of speech information, $R_{s} T$ bits, in each packet. There is an optimum value for frame duration because reducing frame duration means that overhead bits will be the dominant, this overhead will consume excessive channel resources. Also, reducing frame duration reduces number of slots $N$ per frame (1). This reduction in the number of slots per frame increases number of collisions because all terminals try to contend on small number of slots. Increasing frame duration also leads to some other problems, increasing frame duration decreases the buffer size which in turn leads to excessive packet drooping (4). Buffer size, $B$, actually representing the number of time slots, $D$, which the packet could live in the terminal transmitting buffer. Each speech terminal contains a firstin first-out buffer to store packets that waiting successful transmission. Each packet in the buffer has a counter that records its age in terms of number of slots; if the packet's age exceeds $D$ slots then this packet must be dropped from the buffer. Therefore, increasing packet duration will decrease packet opportunity for transmission.

$$
D=\left\lceil\frac{D_{\max } N}{T}\right\rceil
$$


Figure 6 shows the effect of frame duration on maximum number of simultaneous conversations with $p_{\text {drop }} \leq$ 0.01. PRMA with random contention scheme is very sensitive to frame duration. Increasing frame duration decreases number of simultaneous conversations. With large frame duration, number of time slots, $D$, that needed for a packet to stay in buffer is decreased and be less than the average packet delay, $4 \mathrm{msec}$, this reduction in $D$ causes the reduction in maximum number of simultaneous conversations. PRMA with permission contention face the same scenario but its sensitivity to frame duration is less.

Normalized capacity, $\eta$, is used as a measure for comparing different PRMA configurations. Number of simultaneous channels in TDMA system is adopted as a normalized measure [1]. In perfect TDMA system with no overhead, the number of voice channels is $R_{c} / R_{s}$ which is equivalent to 22.5 (using the proposed parameter values). Normalized capacity, $\eta$, is defined as:

$$
\eta=\frac{M_{0.01}}{R_{c} / R_{s}}
$$

Figure 7 shows the normalized capacity under different number of channels ${ }^{1}$, simulation run for different values of transmission rates, $R_{c}$. Increasing the transmission rate increases the normalized capacity. But this increase in normalized capacity is bounded to 1.64; PRMA cannot reach its upper bound ${ }^{2}, 2.09$, due to the effects of wasted time slots. Both, random and permission contention schemes, show the same results. But at low traffic rates random contention scheme gives better capacity than permission scheme. This improvement in capacity at low traffic load is due to utilizing most of available slots, number of collisions in random contention at low traffic rates is smaller than that in permission scheme and therefore, number of wasted slots is reduced.

In PRMA, packets that stay in terminals transmitting buffers for a time greater than $D_{\max }$ will be dropped because voice applications cannot tolerate transmission delays. Figure 8 displays the relationship between delay limit, $D_{\max }$, and the number of simultaneous con- versation. For low packet delay limits, most of generated packets are dropped from the buffer. Both contention schemes are sensitive to packet delay limit. In random contention scheme most of the generated packets are dropped from the terminal buffers with low $D_{\max }$, when $D_{\max }<0.004 \mathrm{~s}$ all generated packets are dropped from the terminal transmitting buffers because the average packet delay is $0.004 \mathrm{~s}$ as shown in Figure 4, therefore, for good

\footnotetext{
${ }^{1}$ Number of channels is equivalent to TDMA capacity, $R_{\mathrm{c}}$ is changed from $64 \mathrm{kbps}$ to $960 \mathrm{kbps}$, and therefore, number of channels is ranged between from 2 to 30 .

${ }^{2}$ Upper bound could be reached in the ideal conditions when there are no wasted slots. upper bound is $N .\left(R_{s} / R_{c}\right)\left(\left(t_{1}+t_{2}\right) / t_{1}\right)$.
}

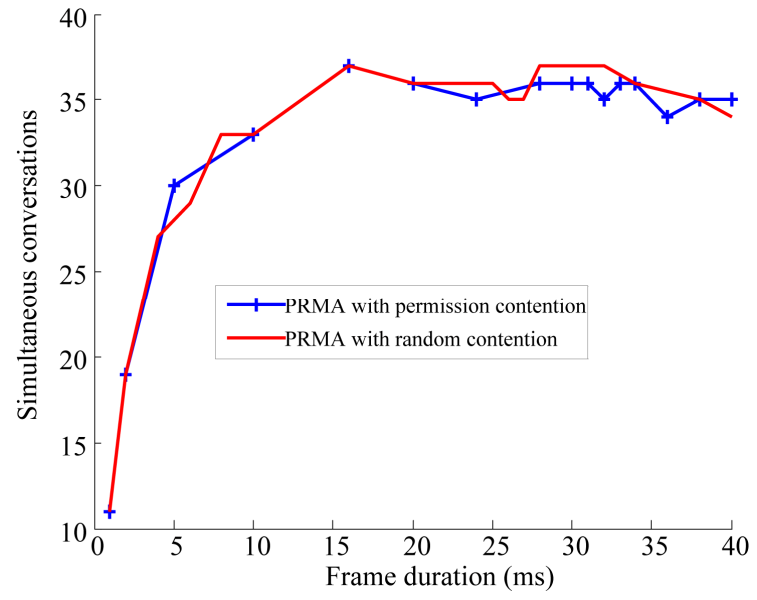

Figure 6. Number of simultaneous conversation with $p_{\text {drop }} \leq$ 0.01 as a function of frame duration.

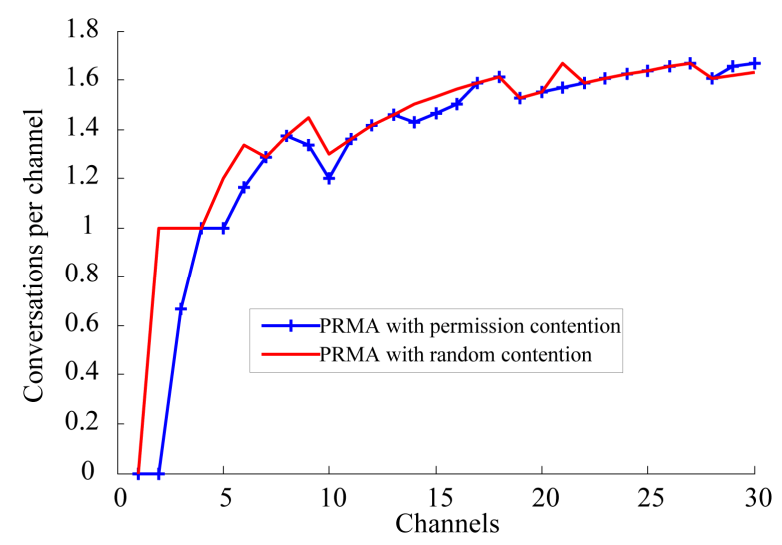

Figure 7. Number of simultaneous conversations per channel as a function of the number of channels.

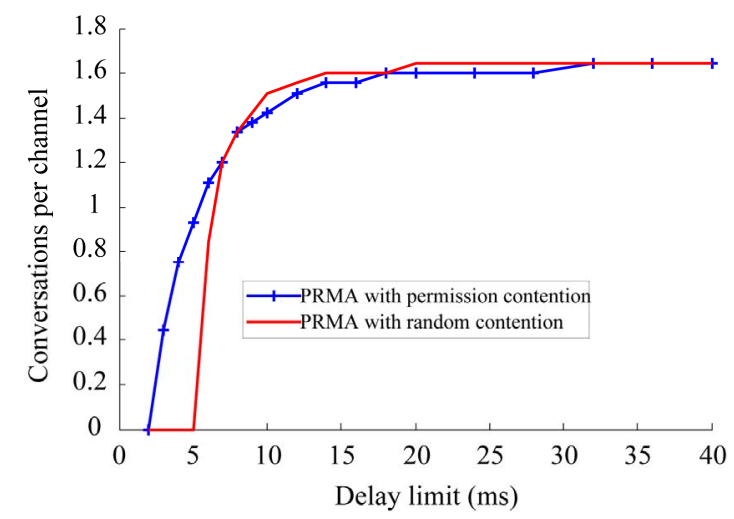

Figure 8. Conversation per channel as a function of speech packet delay limit.

system capacity, $D_{\max }$ must be adjusted carefully to avoid this excessive packet drooping. Permission contention scheme also cannot deal with low packet delay limits, as shown in the Figure 8 normalized system capacity under permission contention scheme at low packet delay limit 
is less than 1.

\section{Conclusions}

In this work we propose a new contention scheme, random contention scheme, for PRMA system and we examined the influence of large number of system variables on PRMA performance. Our results are compared with PRMA system under permission scheme.

Simulation results show that with our new scheme we could reach the maximum number of simultaneous conversation per channel as in PRMA under permission contention scheme. Our approach gives better results in some cases. The main features of our approach are: reducing average packet delay at high traffic rates and reducing number of wasted slots for low traffic rates. Simulation results show that for high traffic cases, number of collisions increased rapidly, this rapid increase in number of collisions is due to small number of available time slots compared to the number of active terminals. These results show that the new contention scheme could be improved by introducing some dynamic contention policy for high traffic rate cases. Dynamic contention policy will increase system capacity by reducing the number of collisions.

\section{References}

[1] S. Nanda, D. J. Goodman and U. Timor, "Performance of PRMA: A Packet Voice Protocol for Cellular Systems," IEEE Transactions on Vehicular Technology, Vol. 40, No. 3, August 1991, pp. 584-598. doi:10.1109/25.97513

[2] D. J. Goodman, R. A. Valenzuela, K. T. Gayliard and B. Ramamurthi, "Packet Reservation Multiple Access for Local Wireless Communications," IEEE Transactions on Communications, Vol. 37, No. 8, August 1989, pp. 885890. doi: $10.1109 / 26.31190$
[3] D. J. Goodman and S. X. Wei, "Efficiency of Packet Reservation Multiple Access," IEEE Transactions on Vehicular Technology, Vol. 40, No. 1, February 1991, pp. 170-176. doi:10.1109/25.69985

[4] S. Nanda, "Analysis of PRMA: Voice Data Integration for Wireless Networks," Proceedings of IEEE Global Telecommunications Conference, San Diego, December 1992, pp. 1984-1988.

[5] F. Barcelo and A. Ramon, "Minislotted Packet Reservation Multiple Access Protocol for Integrated Voice-Data Transmission," Proceedings of Singapore ICCS '94 Conference, Singapore, Vol. 1, 14-18 November 1994, pp. 320-323.

[6] J. -H. Wen and J.-W. Wang, "A New Protocol For Wireless Voice Communications-Non-Collision Packet Reservation Multiple Access," Proceedings of the 6th IEEE International Symposium on Personal Indoor and Mobile Radio Communications, Toronto, Vol. 2, 27-29 September 1995, pp. 638-642. doi:10.1109/PIMRC.1995.480946

[7] G. Pierobon, A. Zanella and A. Salloum, "ContentionTDMA Protocol: Performance Evaluation," IEEE Transactions on Vehicular Technology, Vol. 51, No. 4, July 2002, pp. 781-788. doi:10.1109/TVT.2002.1015358

[8] J.-H. Wen, Y.-S. Chen, T.-S. Chang and J.-K. Lain, "Theoretical Analysis and Performance of NC-PRMA Protocol for Multichannel Wireless Networks," Wireless Personal Communications, Vol. 39, No. 2, 2006, pp. 199-213. doi:10.1007/s11277-006-9088-8

[9] A. Al-Shanyour, A. A. Mahmoud, T. Sheltami and S. A. AlQahtani, "Packet Reservation Multiple Access (PRMA) with Random Contention," Proceedings of ACS/IEEE International Conference on Computer Systems and Applications, Amman, 13-16 May 2007, pp. 158-164.

[10] N. Wattanamongkhol, W. Srichavengsup, P. Vara-urairat, S. Siwamogsatham and L. Wuttisiittikulkij, "Analysis of a Multiple-Token Contention Scheme for Broadband Wireless Access Networks," Proceedings of IEEE International Conference on Communications Workshops, Beijing, 19-23 May 2008, pp. 68-72. doi:10.1109/ICCW.2008.18 